\title{
Claustrum, consciousness, and time perception
}

\author{
Bin Yin ${ }^{1}$, Devin B Terhune ${ }^{2}$, John Smythies ${ }^{3}$, and Warren H Meck ${ }^{1}$
}

\section{Addresses:}

${ }^{1}$ Department of Psychology and Neuroscience, Duke University, Durham, NC, USA

${ }^{2}$ Department of Psychology, Goldsmiths, University of London, London, UK

${ }^{3}$ Center for Brain and Cognition, University of California, San Diego, La Jolla, CA US 


\begin{abstract}
The claustrum has been proposed as a possible neural candidate for the coordination of conscious experience due to its extensive "connectome". Herein we propose that the claustrum contributes to consciousness by supporting the temporal integration of cortical oscillations in response to multisensory input. A close link between conscious awareness and interval timing is suggested by models of consciousness and conjunctive changes in meta-awareness and timing in multiple contexts and conditions. Using the striatal beat-frequency model of interval timing as a framework, we propose that the claustrum integrates varying frequencies of neural oscillations in different sensory cortices into a coherent pattern that binds different and overlapping temporal percepts into a unitary conscious representation. The proposed coordination of the striatum and claustrum allows for time-based dimensions of multisensory integration and decision-making to be incorporated into consciousness.
\end{abstract}

\title{
Introduction
}

Consciousness is not a unitary phenomenon, but a class of states that can be viewed as distributed along a continuum of arousal or awareness ranging from none to full awareness [1, 2, $\left.3^{\bullet}\right]$. Conscious awareness varies considerably within an individual across different contexts, such as non-conscious (or minimally-conscious) states as in non-REM sleep to fully conscious states as in normal wakefulness [4]. The identification of the neural correlates of consciousness is both an enduring challenge in consciousness science and the focus of much research although our understanding of how the brain enables conscious states and how shifts in brain states contribute to fluctuations in consciousness remains in its infancy [5]. Emerging evidence from studies of awareness across time, alterations in meta-awareness involving changes in neural synchronization, and clinical populations characterized by distortions in awareness, suggest a close intersection between conscious states and interval timing. Consequently, temporal integration mechanisms have been implicated in the neural substrates of consciousness [6, 7]. Moreover, the primary 
neurophysiological correlates of consciousness in these studies has been neural synchronization as a function of alternation between phasic and sustained activity [8, 9•, 10, 11, 12].

In recent years, the claustrum was proposed as a possible neural candidate for the coordination of conscious awareness $[13,14]$ and to play a key role in integrating diverse sources of neural information during the formation of unified conscious percepts $[15,16]$. The interhemispheric connections of the claustrum enable the coordination of bilateral cortical functions by way of its ipsilateral and contralateral connections with prefrontal, premotor, and motor areas. Working from the starting point that subjective time constitutes the "infrastructure of consciousness" [17] we propose that the claustrum plays a crucial role in consciousness by supporting the temporal integration of cortical and thalamic oscillations involved in the multiplexing of sensory input used for interval timing and working memory [18•].

The continuity of experience transduced by temporal integration is one of the defining features, if not the defining feature, of consciousness [19]. Accordingly, a first step in addressing the relevancy of time in consciousness requires a re-evaluation of what is meant by "conscious processing" and the control of an internal clock $[20 \bullet, 21]$. On closer examination, a process might be said to be "conscious" in three distinct senses.

(a) one is aware of the process

(b) the operation of the process is accompanied by awareness (of its results) and (c) awareness enters into or causally influences the process.

Crick and Koch provided an outline for the scientific study of consciousness [15]. In this framework, the authors proposed that an alternative to tackling the "hard problem" of qualia [22] would be to identify some neural correlate(s) of consciousness in causal terms, that is, "finding a minimal set of neuronal events that gives rise to a specific aspect of a conscious percept". As a consequence, they focused exclusively on neural activity related to a specific sensory modality, i.e. the visual system of primates, leaving unexplored other aspects of consciousness, such as emotion and self-awareness. Although we, in principle, agree with Crick and Koch's perspective, we maintain 
that consciousness science should investigate how inputs from different sensory channels can emerge as a complete picture of our ever-changing conscious experience. As a consequence, any specific sensory percept is construed as one input to this emergent principle of coalition. In other forums, this has traditionally been referred to as the 'neural binding' problem [23].

It is easily overlooked that humans share with other animals a remarkable ability to estimate the durations of events and subjectively experience a sense of time passing [24]. It is also tempting to assume that the experience of conscious states, and in particular self-awareness, is dependent upon the ability to perceive duration and to understand the concepts of past, present, and future [25, but see 26] which leads researchers to face the dilemma of whether non-human animals have human-like conscious experience. Although performing a classic timing task such as the peakinterval procedure $[27,28]$ may not require complete awareness of time passing, more fundamentally our conscious experience may actually be organized by an underlying timing mechanism. Indeed, distinct from physical entities that have multiple dimensions (at least three dimensions in space and one dimension in time in classical physics), time is arguably the only dimension for mental entities (e.g., thought, feeling, sensory perception, etc.), unless they have other dimensions that could only be measured in a phenomenal world [13]. Therefore, it is intriguing to question whether our subjective experiences coalesce mainly because we have a built-in temporal integration process that coordinates different channels of inputs into uniform subjective states. This is particularly important not only because of the "hard problem" of consciousness that has lingered for centuries, but also because timing is disrupted in various mental disorders [29] that can be regarded as "disorders of conscious experience" and thereby may prove valuable in elucidating basic mechanisms and developing treatments. One example is schizophrenia, because schizophrenic patients have a distorted sense of reality and temporal structure [30, 31]. Another example is pathological gambling, because pathological gambling can be directly associated with an altered state of consciousness, dysfunctional risk assessment, and a skewed perception of time and rate of return [32]. The observation that pathological gamblers exhibit reduced gamma 
synchronization in paralimbic cortical structures during rest as well as an impairment in task-related changes compared to controls has been associated with a loss of conscious coherence [33]. Behavioral studies suggest that gamblers may be ultra-sensitive to time and experience a delusional high rate of return in various aspects of their lives [34]. Consequently, disrupted optimization of timing abilities and impaired self-awareness may contribute to compulsive gambling behavior [32, $34,35 \bullet \cdot]$.

\section{Consciousness, metacognition and interval timing}

By definition, consciousness refers to awareness of one's unique thoughts, feelings and sensations of the environment. A key characteristic is that these experiences are constantly shifting. The ever-shifting stream of thoughts can change dramatically from one moment to the next, but one's experience of it seems smooth and effortless. How does the brain enable such continuity of experience and what are the essential mechanisms for the emergence of conscious experience? Three possible criteria can be derived from 'higher-order' theories of consciousness [36] and 'integrated information theory' [37]:

1. The ability to select one state out of the indefinite possibilities (differentiated information) e.g., differences between a light sensor and a conscious agent.

2. The ability to have awareness of mental representations (metacognition; second-order representations).

3. The ability to tag personal meaning to the state.

It is important to note that there exist fundamental differences between sensitivity (non-conscious) and awareness (conscious): sensitivity relies on the first-order representation in the system, whereas awareness relies on the second-order representation in the system. That is, sensitivity entails the ability to respond in specific ways to certain states of affairs, whereas awareness requires the agent to have the knowledge of the fact that she or he is sensitive to some state of affairs and also cares about a certain state of affairs. For example, a camera doesn't lack consciousness because it's only sensitive to light, but because it has no awareness of being sensitive to light. One 
could only make the camera conscious by enabling a second-order mechanism that could coordinate its moments of recording light with its memory of past recordings of light, as well as its own preference for that particular moment of light-sensing and memory traces - this mechanism could obviously be the time-keeping mechanism described above. For such a higher-order timing mechanism to work, each channel of inputs must have its own clock(s) so that these clock phases can be synchronized to form a representation of the present — "now". The proposal that we present in this review is that the claustrum is critical for the type of temporal integration required by consciousness.

A further link between metacognition and interval timing is suggested by psychological manipulations that alter conscious states and distort time perception as well as by disorders of consciousness. Two such psychological techniques include hypnosis, which involves the administration of a hypnotic induction, involving suggestions for reduced awareness and metaawareness [38], and meditation, which involves a variety of practices with the intent to foment awareness of mental representations [39]. A hypnotic induction reliably produces a tendency to underestimate time, particularly in highly suggestible individuals $[6,8]$, who comprise approximately $10-15 \%$ of the population [38]. Alongside distortions in time perception, highly suggestible individuals routinely experience states of depersonalization and derealization spontaneously following a hypnotic induction [39]. Although they have not been systematically studied, depersonalization disorder patients are similarly known to experience pronounced disruptions in time perception [39] and drugs that elicit distortions in time perception also induce depersonalization [41]. In contrast, preliminary evidence suggests that meditation training produces a tendency to overestimate intervals [42].

The mechanisms underlying these distortions are not well understood but may lie in atypical metacognition in these populations. A common theme in models of hypnosis is that highly suggestible individuals are characterized by impaired executive monitoring and that the ability to respond to hypnotic suggestions is facilitated by reduced awareness of intentions underlying 
responses [38, 43]. Following a hypnotic induction, highly suggestible individuals exhibit reduced medial prefrontal cortex activity [44] as well as reduced frontal-parietal alpha [8] and frontal-centralcentral beta synchrony [45] relative to low suggestible individuals. These changes implicate default mode, executive attention, and motor networks involved in metacognition, cognitive control, and interval timing [46]. In contrast, there is evidence suggesting that meditators have greater awareness of their intentions to act [39]. Cumulatively, these results suggest that awareness of mental representations is closely intertwined with one's perception of time.

\section{Striatal Beat Frequency (SBF) model of interval timing}

In the striatal beat frequency (SBF) model of interval timing $[29,47,48,49,50]$ duration estimation is based upon the coincidence detection of oscillatory processes in cortico-striatal circuits. The SBF model supposes that at the onset of a "to be timed" signal, populations of cortical (and thalamic) neurons phase reset (and synchronize) and begin oscillating at their endogenous periodicities. Dopamine release from the ventral tegmental area at the onset of the signal is believed to play a part in this resetting function for cortical neurons while also acting as a 'start gun', and dopamine release from the substantia nigra pars compacta at signal onset works in a similar fashion to reset the weights of the synaptic connections in the dorsal striatum [51]. The detection of coincident activation of specific cortical oscillatory patterns is the role of striatal medium spiny neurons (MSNs). The adjustment of cortico-striatal synaptic weights allows the MSNs to discriminate and become 'tuned' to specific patterns of coincident oscillatory activity, thus increasing their likelihood of firing upon similar patterns of cortical activation in the future. This property accounts for the close correspondence between interval timing and working memory performance, which are held to depend on the same neural representation of a specific stimulus [18•]. Given that oscillatory activation repeats itself at regular intervals (its period) and changes in a systematic manner as a function of time (its phase), these cortical oscillatory patterns, when observed across neurons differing in their intrinsic periodicity, can represent time intervals in the seconds-to-minutes range although their neural firing occurs in the milliseconds range. The MSNs are able to detect these 
patterns, which bear resemblance to musical chords, by acting as coincidence detectors or 'perceptrons' [52]. Striatal output travels to the thalamus along two pathways: the direct (dopamine $D_{1}$ receptor-mediated) and indirect (dopamine $D_{2}$ receptor-mediated), then loops back to the cortex and striatum, influencing the rate of oscillatory activity and permitting alterations in clock speed by changing the input to MSNs [53]. Differential activity in the direct and indirect pathways of the basal ganglia may serve to start, stop, or reset the timing process [49]. Consequently, the SBF model has the advantage of being consistent with the known psychophysics, neuropharmacology, and neuroanatomy of interval timing while at the same time making testable predictions regarding the functioning of its components $[20 \bullet, 48,51,54,55,56]$. A unified timing model that incorporates the SBF model of beat-based timing using cortico-striatal circuits and duration-based models using olivocerebellar circuits is outlined in Figure 2. This model is based on coordinated activity in the core striatal and olivocerebellar networks that are interconnected with each other and the cerebral cortex through multiple synaptic pathways [20•, 57].

\section{Claustrum and consciousness}

Crick and Koch described how the claustrum might play a role in integrating separate sensations into the unitary percepts that we experience as consciousness [16]. Smythies and colleagues [13, 14, 58, 59••] subsequently presented a more detailed hypothesis about how such temporal integration might occur based on higher-order temporal synchronization and the neuroanatomy of the claustrum. The claustrum is broadly divided into 3 sub-regions, the anterior-dorsal region connected with somatosensory and motor cortices, a posterior dorsal region connected with the visual cortex, and a ventral area connected to the auditory cortex $[60,61,62 \bullet \bullet, 63,64,65 \bullet$. The claustrum has reciprocally distributed projections to virtually all regions of the cortex (e.g., frontal, premotor, ventral anterior cingulate, ventral temporal, visual, motor, somatosensory, olfactory, and entorhinal cortex), as well as sub-cortical structures (e.g., caudate nucleus, putamen, globus pallidus, and lateral amygdala) that are illustrated in Figure 1. These extensive bilateral and 
interclaustral connections support the hypothesis that the claustrum serves as a 'synchrony detector', thereby coordinating information sharing and binding throughout the brain $[13,16]$.

White et al. [66] have recently reported unanticipated findings relating to the anatomy of connections between the claustrum and the cortex. These include the observation that the projections from noncingulate cortex to the claustrum are discrete, sparse and mainly directed to the contralateral claustrum while projections from the claustrum to the non-cingulate cortex are discrete, sparse and directed to the ipsolateral cortex. In contrast, the cingulate cortex projects mainly to the contralateral claustrum and the claustrum projects to the ipsolateral cortex as above. The connections between the claustrum and the cingulate, however, are both diffuse and massive with discrete regions in the cingulate cortex projecting to most of the claustrum and vice versa. These anatomical connections suggest that the main output of the claustrum to the motor-control system doesn't go via the sparse direct route, but via the dense route with a relay in the cingulate cortex. This allows the claustrum to play a modulatory role in all the many functions of the cingulate cortex besides controlling motor output and behavior via the premotor cortex. These findings also require an extension of the proposed functions of the claustrum as outlined by Smythies and colleagues [67].

Although other brain areas have extensive interconnections (e.g., amydala, parietal cortex, thalamus), one way in which the claustrum is distinctive is in the proportion of claustral-cortical neurons that use synaptic zinc. Traditionally, $\mathrm{Zn+}$ neurons have been associated with hippocampal mossy fibers and the generation of synchronized oscillations associated with activity-dependent neuroplasticity. The relatively dense zinc-positive $(\mathrm{Zn+})$ terminations in the claustrum suggest the ability to coordinate multisensory processes more effectively given that synaptically released zinc is thought to control a 'window' of postsynaptic excitability without altering firing rates $[68,69]$.

Cortico-claustral-cortico circuits provide for strong feed forward inhibitory (FFI) processes. An FFI is composed of a group of pre-synaptic neurons that directly excite both glutamaterigic excitatory and GABAergic inhibitory interneurons and provide greater synaptic input to the latter [70]. The postsynaptic neurons are interconnected and circuits that lack inhibition simply relay pre-synaptic activity 
to post-synaptic neurons. In contrast, post-synaptic neurons in an FFI are highly sensitive to the relative timing of action potentials, and this allows for the modulation of neural synchrony as transmitted by the pre-synaptic neurons [59.•]. Neuromodulators and feedback connections may modulate the temporal sensitivity of such circuits and gate the propagation of synchrony into other layers as well as sub-cortical and cortical areas [58]. The prevalence of strong FFI circuits throughout the brain suggests that synchrony codes in conjunction with time-sensitive corticothalamic-basal ganglia (CTBG) circuits are the basis for the temporal integration required for consciousness. In this manner, the claustrum is able to support the full continuum of consciousness through the coordination of CTBG timing circuits in conjunction with cortical-striatal-hippocampalinsular networks, thereby creating a timing-based conscious experience [72, 73••].

This hypothesis also suggests that the claustrum plays an important executive function. Our proposal is that decision-making is mediated by select assemblies of neurons, with synchronized oscillations at different gamma frequencies, that carry integrated sensory information modulated by saliency (reinforcing) mechanisms. These assemblies compete on a winner-take-all basis for access to the output from the claustrum to the prefrontal and premotor cortices. In this way the winner directs the voluntary decision-based behavior of the subject. Exact timing of these events plays a crucial role in the system.

Goll and colleagues [74••] have recently proposed a hypothesis that the claustrum is closely involved in selective attention. They hypothesize that a widespread top-down input from the prefrontal cortex to the claustrum competitively modulates the output from the claustrum to various cortical localities. This allows the claustrum initially to process many different sensory objects simultaneously, and then to allow just one of these at any moment to constitute the center of attention with the inhibition of its competitors. The claustrum is further subject to down-up modulation by unexpected stimuli from sensory cortices that can over-ride the selective attention strategy. The claustrum also has a projection to the motor cortex including the frontal eye fields that promote motor movements, such as eye and neck movements, which modulate the focus of 
attention and has been likened to a mechanism for moving the 'spotlight' of attention.

Some recent evidence has cast doubt on the role of the claustrum in consciousness [75]. Combat veterans with penetrating traumatic brain injuries were studied in terms of the effects of claustrum lesions and loss of consciousness on long-term cognitive functions. The extent of claustrum damage was associated with the duration, but not the frequency, of loss of consciousness. This could be interpreted as indicating that the claustrum plays an important role in regaining consciousness, but not in maintaining cognitive function. In contrast, other researchers have reported that selective bilateral lesions of the claustrum and external capsule resulting from herpes simplex encephalitis and Sugihiritake mushroom poisoning leads to severe encephalopathy with disruptions in consciousness, psychotic symptoms and seizures [62••]. A specific role for the claustrum in auditory scene analysis as reflected by sensitivity to sensory change has also been identified [76]. Cogent to the current hypothesis, Wittmann and colleagues [77] assessed the effect of size and localization of brain lesions on the perception of the temporal order of two acoustic stimuli. Although a moderate association of lesion size and temporal-order threshold was observed among all brain-lesions patients, a clear temporal discrimination deficit was specifically observed in those patients with damage to the claustrum. Unfortunately, no assessment of consciousness was made in this study; hence correlations between temporal processing and consciousness couldn't be performed. Similar types of impairments in temporal order and spatial reversal learning have been observed in rats following lesions of the anterior claustrum [78]. Moreover, the impairments in duration discrimination produced by lesions of the dorsal striatum were magnified as a function of the extent of collateral damage to the claustrum [79].

In support of the role of the claustrum in timing and time perception, a meta-analysis of fMRI studies [80] showed activation of the claustrum during the timing of supra-second durations, particularly in reproduction tasks. Subsequent neuroimaging studies have shown activation of the insula/insular cortex (a region closely related to the claustrum) in a variety of timing tasks [81, 82]. Moreover, Koubeissi et al. [83] recently reported a case study in which the researchers were 
apparently able to turn consciousness "on and off" by stimulating a small area of the claustrum/insular cortex.

\section{Conclusions}

We endorse the 'attention hypothesis' for claustrum function [74••], which can readily be combined with the SBF model whereby the claustrum serves an executive function for the selection of the stimuli to be processed within the multiplexing system of interval timing and working memory [18•]. In this manner, the claustrum is able to modulate synchronized oscillations from widely distributed cortical regions in order to determine which patterns of spike coincidence-detection should be processed by cortico-striatal-thalamo-cortical timing circuits - either individually (selective attention) or in parallel (divided attention). The anatomical details and attentional functions of this type of timesharing system have been previously described [74・•], but the specific neurophysiological mechanisms remain to be delineated. The basis for this mechanism is suggested by recent evidence indicating the role of competitive synchronized gamma oscillations organized by the Pearson mechanism [59••]. In addition, we propose that the motor output from the claustrum mediates the selection of all voluntary behaviors as well as the movements modulating the focus of attention [67]. Future experimental work addressing the relation between self and time [84] should be designed to test these predictions regarding duration and temporal order discrimination as well as attentional time-sharing and simultaneous temporal processing $[85,86,87]$ using optogenetic and designer receptor exclusively activated by designer drug (DREADD) techniques within the claustrum and CTBG $[62 \bullet \bullet, 88,89,90]$. Such studies should allow for the dissection of beat-based versus intervalbased timing [57] within temporal windows defining past, present, and future states of awareness $[91,92,93]$ while taking into account the flow of time from temporal sensation to time perception and production in the service of consciousness and the relation between time and self $[84,94,95,96$, 97••]. 


\section{Acknowledgements}

We thank Melissa Allman, Bon-Mi Gu, Tadeusz Kononowicz, Nicholas Lusk, Trevor Penney, and Elijah Petter for valuable discussions concerning the topic of consciousness and time perception. DBT was supported by Bial Foundation bursary 144/14. 


\section{References and recommended reading}

Papers of particular interest, published within the period of review, have been highlighted as:

- of special interest

$\bullet \bullet$ of outstanding interest

1. Churchland PS: Reduction and the neurobiological basis of consciousness. In Consciousness in Contemporary Science. Marcel AJ, Bisiach E (Eds.), Oxford: Oxford University Press; 1992: 273-304.

2. Mormann F, Koch C: Neural correlates of consciousness. Scholarpedia 2007, 2(12):1740.

3. Anzulewicz A, Asanowicz D, Windey B, Paulewicz B, Wierchoñ M, Cleeremans A: Does

- level of processing affect the transition from unconscious to conscious perception? Conscious Cogn 2015, 36:1-11.

Tests the "level of processing" (LoP) hypothesis that the transition from unconscious to conscious perception is influenced by the level of processing imposed by task requirements. Results from a series of letter classification experiments indicated that low or moderate stimulus visibility was reported more frequently in the low-level task than the high-level task. These data suggest that the transition from unconscious to conscious perception is more or less gradual depending on the level of processing required to identify and categorize letters as being consonants or vowels with lowercase or uppercase letters.

4. Casali AG, Gosseries O, Rosanova M, Boly M, Sarasso S, Casali KR, Casarotto S, Bruno MA, Laureys S, Tononi G, Massimini M: A theoretically based index of consciousness independent of sensory processing and behavior. Sci Transl Med 2013, 5(198):198ra105.

5. Tsuchiya N, Wilke M, Frässle S, Lamme VAF: No-report paradigms: extracting the true neural correlates of consciousness. Trends Cogn Sci 2015, 19(12):757-770.

6. Naish P: The perception of time in hypnosis. Subjective time: the philosophy, psychology, and neuroscience of temporality. Lloyd D, Arstila V (Eds.), MIT Press, 2014, 621-636.

7. Meck WH, Vatakis A, van Rijn H: Timing \& Time Perception Reviews: opening the door to theoretical discussions of consciousness, decision-making, multisensory processing, time cells and memory mapping ... to name but a few issues of relevance to temporal cognition. Timing Time Percept Rev 2014, 1(1).

8. Terhune $D B$, Cardeña $E$, Lindgren M: Differential frontal-parietal phase synchrony during hypnosis as a function of hypnotic suggestibility. Psychophysiology 2011, 48: 1444-1447.

9. Crone JS, Schurz M, Höller Y, Bergmann J, Monti M, Schmid E, Trinka E, Kronbichler M:

- Impaired consciousness is linked to changes in effective connectivity of the posterior cingulate cortex within the default mode network. Neurolmage 2015, 110:101-109.

Applied spectral dynamic causal modeling to assess effective connectivity within the default mode network in patients with disorders of consciousness. Results indicated that the degree of self-inhibition and strength of oscillation in the posterior cingulate cortex is correlated with the level of consciousness.

10. Doesburg SM, Green JJ, McDonald JJ, Ward LM: Rhythms of consciousness: binocular rivalry reveals large-scale oscillatory network dynamics mediating visual perception. PLOS ONE 2009, 4(7):e6142

11. Tononi G, Koch, C: Consciousness: here, there and everywhere? Philos Trans $R$ Soc $B$ 2015, 370:20140167.

12. Ward LM: The thalamic dynamic core theory of conscious experience. Conscious Cogn 2011, 20:464-486.

13. Smythies J, Edelstein L, Ramachandran V: Hypotheses relating to the function of the claustrum. Front Integr Neurosci 2012, 6:53.

14. Smythies J, Edelstein L, Ramachandran V: The functional anatomy of the claustrum: the net that binds. WebmedCentral NEUROSCIENCES 2012, B(3):WMC003182

15. Crick FC, Koch C: A framework for consciousness. Nat Neurosci 2003, 6:119-126. 
16. Crick FC, Koch $\mathrm{C}$ : What is the function of the claustrum? Philos Trans $R$ Soc $B$ Lond 2005, 360:1271-1279.

17. Zahavi D: Self-awareness and alterity. Evanston, IL: Northwestern University Press. 1999.

18. Gu B-M, van Rijn H, Meck WH: Oscillatory multiplexing of neural population codes for

- interval timing and working memory. Neurosci Biobehav Rev 2015, 48:160-185.

Describes a multiplexing model explaining how interval timing and working memory can originate from the same oscillatory processes, but differ in terms of which dimension of the neural oscillation is utilized for the extraction of item, temporal order, and duration information. This hybrid model is based upon the striatal beat frequency model of interval timing originally proposed by Matell and Meck [47].

19. Bodovitz A: Consciousness disintegrates without conscious vectors. Med Hypoth 2008, 70:8-11.

20. Allman MJ, Teki S, Griffiths TD, Meck WH: Properties of the internal clock: first- and

- $\quad$ second-order principles of subjective time. Annu Rev Psychol 2014, 65:743-771.

Provides an extensive overview of clock speed, memory translation, timescale invariance, multisensory integration, and attentional time-sharing for interval timing in the seconds to minutes range.

21. Velmans M: Is human information processing conscious? Behav Brain Sci 1991, 14(4):651-669.

22. Howell RJ, Alter T: Hard problem of consciousness. Scholarpedia 2009, 4(6):4948.

23. Revonsuo A: Binding and the phenomenal unity of consciousness. Conscious Cogn 1999, 8:173-185.

24. Lloyd D, Arstila V: Subjective time: the philosophy, psychology, and neuroscience of temporality. MIT Press, 2014.

25. Allman MJ, Yin B, Meck WH: Time in the psychopathological mind. Subjective time: the philosophy, psychology, and neuroscience of temporality. Lloyd D, Arstila V (Eds.), MIT Press, 2014, 637-654.

26. Meyer U: Consciousness and the present. Y Dolev, M Roubach (eds.), Cosmological and Psychological Time, Boston Studies in the Philosophy and History of Science 2016, 285:143153.

27. Church RM, Meck WH, Gibbon J: Application of scalar timing theory to individual trials. J Exp Psychol Anim Behav Process 1994, 20:135-155.

28. Rakitin BC, Gibbon J, Penney TB, Malapani C, Hinton SC, Meck WH: Scalar expectancy theory and peak-interval timing in humans. J Exp Psychol Anim Behav Process 1998, 24:15-33.

29. Allman MJ, Meck WH: Pathophysiological distortions in time perception and timed performance. Brain 2012, 135:656-677.

30. Ciullo V, Spalletta G, Caltagirone C, Jorge RE, Piras F: Explicit time deficit in schizophrenia: systematic review and meta-analysis indicate it is primary and not domain specific. Schizophr Bull in press. doi:10.1093/schbul/sbv104

31. Martin B, Wittmann M, Franck N, Cermolacce M, Berna F, Giersch A: Temporal structure of consciousness and minimal self in schizophrenia. Front Psychol 5:1175.

32. Brevers D, Cleeremans A, Bechara A, Greisen M, Kornreich C, Verbanck P, Noël X: Impaired self-awareness in pathological gamblers. J Gambling Stu 2013, 29:119-129.

33. Thomsen KR, Joensson M, Lou HC, Møller A, Gross J, Kringlbach ML, Changeux J-P: Altered paralimbic interaction in behavioral addiction. Proc Natl Acad Sci USA 2013, 110:4744-4749.

34. Wiehler A, Bromberg U, Peters J: The role of prospection in steep temporal reward discounting in gambling addiction. Front Psychiatry 2015, 6:112.

35. Wittmann M: The inner sense of time: how the brain creates a representation of

-• duration. Nat Rev Neurosci 2013, 14:217-223.

Describes the idea that temporal processing is mediated by climbing neural activity in the insular cortex. This activation is associated with the temporal integration across many body systems, including feeling states and 
multisensory thought processes that may be coordinated by the claustrum. Self-awareness of this inner sense of time potentially links consciousness to time perception.

36. Rosenthal D, Werisberg J: Higher-order theories of consciousness. Scholarpedia 2008, 3(5):4407.

37. Tononi G: Integrated information theory. Scholarpedia 2015, 10(1):4164.

$\bullet$

An up-to-date description of the highly influential (IIT) attempts to identify the essential properties of consciousness (axioms) and, from there, infers the properties of physical systems that can account for it (postulates).

38. Oakley DA, Halligan PW: Hypnotic suggestion: opportunities for cognitive neuroscience. Nat Rev Neurosci 2013,14(8):565-576.

39. Jo HG, Hinterberger T, Wittmann M, Schmidt S: Do meditators have higher awareness of their intention to act? Cortex 2015, 65:149-158.

40. Spiegel D, Lewis-Fernandez R, Lanius R, Vermetten E, Simeon D, Friedman M: Dissociative disorders in DSM-5. Ann Rev Clin Psychol 2013, 9:299-326.

41. Wittmann M, Carter O, Hasler F, Cahn BR, Grimberg U, Spring P, Hell D, Flohr H, Vollenweider $\mathrm{F} X$ : Effects of psilocybin on time perception and temporal control of behaviour in humans. J Psychopharmacology 2007, 21(1):50-64.

42. Kramer RS, Weger UW, Sharma D: The effect of mindfulness meditation on time perception. Conscious Cogn 2013, 22:846-852.

43. Dienes Z, Perner J: Executive control without conscious awareness: the cold control theory of hypnosis. Jamieson GA (Ed.), Hypnosis and conscious states: The cognitive neuroscience perspective Oxford, UK: Oxford University Press. 2007, 293-314.

44. McGeown WJ, Mazzoni G, Venneri A, Kirsch I: Hypnotic induction decreases anterior default mode activity. Conscious Cogn 2009, 18:848-855.

45. Jamieson GA, Burgess AP: Hypnotic induction is followed by state-like changes in the organization of EEG functional connectivity in the theta and beta frequency bands in high-hypnotically susceptible individuals. Front Hum Neurosci 2014, 8:528.

46. Miele DB, Wager TD, Mitchell JP, Metcalfe J: Dissociating neural correlates of action monitoring and metacognition of agency. J Cogn Neurosci 2011, 23:3620-3636.

47. Buhusi CV, Meck WH: What makes us tick? Functional and neural mechanisms of interval timing. Nat Rev Neurosci 2005, 6:755-765.

48. Coull JT, Cheng RK, Meck WH: Neuroanatomical and neurochemical substrates of timing. Neuropsychopharmacology 2011, 36:3-25.

49. Matell MS, Meck WH: Cortico-striatal circuits and interval timing: coincidence-detection of oscillatory processes. Cogn Brain Res 2004, 21:139-170.

50. van Rijn H, Gu B-M, Meck WH: Dedicated clock/timing-circuit theories of time perception and timed performance. Adv Exp Med Biol 2014, 829:75-99.

51. Kononowicz TW: Dopamine-dependent oscillations in frontal cortex index "start-gun" signal in interval timing. Front Hum Neurosci 2015, 9:331.

52. Buhusi CV, Oprisan SA: Time-scale invariance as an emergent property in a perceptron with realistic, noisy neurons. Behav Process 2013, 95:60-70.

53. Oprisan SA, Buhusi CV: Modeling pharmacological clock and memory patterns of interval timing in a striatal beat-frequency model with realistic, noisy neurons. Front Integr Neurosci 2011, 5:52.

54. Hashimoto $Y$, Yotsumoto $Y$ : Effect of temporal frequency spectra of flicker on time perception: Behavioral testing and simulations using a striatal beat frequency model. Timing Time Percept 2015, 3:201-222.

55. Oprisan SA, Buhusi CV: What is all the noise about in interval timing? Phil Trans $R$ Soc $B$ 2014, 369:20120459. 
56. Matthews WJ, Terhune DB, van Rijn H, Eagleman DM, Sommer MA, Meck WH: Subjective duration as a signature of coding efficiency: emerging links among stimulus repetition, prediction coding, and cortical GABA levels. Timing Time Percept Rev 2014, 1(5):1-11.

57. Teki S, Grube M, Griffiths TD: A unified model of time perception accounts for durationbased and beat-based timing mechanisms. Front Integr Neurosci 2011, 5:90.

58. Smythies $\mathrm{J}$ : The nature of consciousness and its relation to brain: The pith of a formidable problem and its possible solution. J Conscious Stud 2014, 21:183-202.

59. Smythies J, Edelstein L, Ramachandran V: Hypotheses relating to the function of the

-• claustrum II: Does the claustrum use frequency codes? Front Integr Neurosci 2014, 8:7. Builds on previous formulations of claustral function by the same authors $[12,13]$ by proposing that the claustrum organizes information processing and conscious awareness utilizing synchronized gamma oscillations operating at three levels: (1) magnification of oscillations in cortico-claustral circuits, (2) integration of these oscillations, and (3) concatenation, spectral processing, and dendritic integration of spike codes within the claustrum.

60. Baizer JS, Sherwood CC, Noonan M, Hof PR: Comparative organization of the claustrum:

- what does structure tell us about function? Front Sys Neurosci 2014, 8:117.

Provides a comparative, evolutionary analysis of the organization of the claustrum in monkeys, great apes, and cetaceans. Anatomical data indicate that the claustrum isn't a continuous structure and that there are many isolated cell islands. This type of organization brings into question the ability of the claustrum to provide "global" integration in support of consciousness. Rather, it provides for independent modulation of distinct claustral-cortical subdivisions and suggests that integration (if it occurs) is done elsewhere. Moreover, the authors offer that the importance of the claustrum for cortical operations may have decreased as cortical complexity increased.

61. Mathur BN: The claustrum in review. Front Sys Neurosci 2014, 8:48.

62. Milardi D, Bramanti P, Milazzo C, Finocchio G, Arrigo A, Santoro G, Trimarchi F, Quartarone

-• A, Anastasi G, Gaeta M: Cortical and subcortical connections of the human claustrum revealed in vivo by constrained spherical deconvolution tractography. Cereb Cortex 2015, 25:406-414.

Constrained Spherical Deconvolution (CSD) tractography allows for the tracking of white matter fibers from cortex to cortical and subcortical targets with subvoxel resolution. Using CSD, this paper provides extraordinary images and analysis of the fibers connecting the claustrim to 4 groups cortical and sub-cortical groups: The anterior and posterior cortico-claustral tracts connecting the claustrum to prefrontal cortex and visual areas; the superior tract linking the claustrum with sensory-motor areas, with the lateral pathway connecting the claustrum to the auditory cortex. In addition, a claustral medial pathway connecting the claustrum with the basal ganglia (e.g., caudate nucleus, putamen, and globus pallidus) was identified as well as extensive bilateral and interclaustral connnections.

63. Smith JB, Alloway KD: Interhemispheric claustral circuits coordinate sensory and motor cortical areas that regulate exploratory behaviors. Front Sys Neurosci 2014, 8:93.

64. Torgerson CM, van Horn JD: A case study in connectomics: the history, mapping, and connectivity of the claustrum. Front Neuroinform 2014, 8:83.

65. Torgerson CM, Irimia A, Goh SYM, van Horn JD: The DTI connectivity of the human

- claustrum. Hum Brain Mapp 2015, 36:827-838.

Using structural and diffusion tensor neuroimaging in healthy human participants this study identified the claustrum as a "primary contributor to global brain network architecture". The authors conclude that the claustral connectome is ideally situated to serve as the putative "gate keeper" for information processing and conscious awareness.

66. White MG, Cody PA, Bubser M, Wang H-D, Deutch AY, Mathur BN: Cortical hierarchy governs rat claustrocortical circuit organization. J. Comp. Neurol. 2016, doi.10.1002/cne.23970.

67. Smythies J: A review of five hypotheses relating to the functions of the claustrum. Proc $R$ Soc $B$ 2016, in press. 
68. Pochwat B, Nowak G, Szewczyk B: Relationship between Zinc (Zn2+) and glutamate receptors in the processes underlying neurodegeneration. Neural Plast 2015, 2015:591563.

69. Rockland KS: Zinc-positive and zinc-negative connections of the claustrum. Front Syst Neurosci 2014, 8:37.

70. Terhune DB, Russi S, Near J, Stagg CJ, Kadosh RC: GABA predicts time perception. $J$ Neurosci 2014, 34:4364-4370.

71. Bruno R: Synchrony in sensation. Curr Opin Neurobiol 2011, 21:701-708.

72. Merchant $\mathrm{H}$, Yarrow $\mathrm{K}$ : How the motor system both encodes and influences our sense of time. Curr Opin Behav Sci 2016, 8:22-27.

73. Yin $\mathrm{B}$, Meck WH: Comparison of interval timing behaviour in mice following dorsal or

-• ventral hippocampal lesions with mice having $\delta$ opioid receptor gene deletion. Philos

Trans R Soc Lond B 2014, 369:20120466.

Demonstrates that the distortions in temporal memory for mice lacking $\delta$-opioid receptors (Oprd1 ${ }^{2 / 2}$ ) are similar to those exhibited by mice with dorsal hippocampal lesions. Earlier studies reported that Oprd $1^{2 / 2}$ mice have altered expression of genes coding for GABA and glutamate transporters, receptors or receptor subunits in the dorsal hippocampus. Within the dorsal striatum, gene expression related to glutamate function was altered in a fashion likely to result in an imbalance between the nigrostriatal and pallidal - striatal pathways. In contrast, the ventral striatum was left largely unchanged. Moreover, previous work using dopamine $D_{1} / D_{5}$ and $D_{2} / D_{3}$ receptor agonists show that the lack of $\delta$-opioid receptors in the Oprd1 ${ }^{2 / 2}$ mice modifies the $D_{1} / D_{5}-$ nigral $/ D_{2} / D_{3}$ pallidal balance in the striatum in favour of the nigral output. These findings are important in establishing a counter-balancing relationship between the dorsal striatum and the dorsal hippocampus in terms of encoding and decoding clock readings stored in temporal memory.

74. Goll Y, Atlan G, Citri A: Attention: the claustrum. Trends Neurosci 2015, 38:486-495.

$\bullet$

Comprehensive review of the input-output connectivity and internal structure of the claustrum. Additional emphasis is placed on the functional role of the claustrum in terms of attention and consciousness and the evaluation of pathologies of the claustrum. A comprehensive model of attention is proposed involving the role of the claustrum in stimulus-driven attention modulation, gain control of sensory processing, and statedependent attentional modulation. The conclusion is that much of the neuromodulation of claustral function remains a mystery.

75. Chau A, Salazar AM, Krueger F, Cristofori I, Grafman J: The effect of claustrum lesions on human consciousness and recovery of function. Conscious Cogn 2015, 36:256-264.

76. Remedios R, Logothetis NK, Kayser C: A role of the claustrum in auditory scene analysis by reflecting sensory change. Front Sys Neurosci 2014, 8:44.

77. Wittmann $M$, Burtscher $A$, Fries $W$, von Steinbüchel N: Effects of brain-lesion size and location on temporal-order judgment. NeuroReport 2004, 15:2401-2405.

78. Grasby K, Talk A: The anterior claustrum and spatial reversal learning in rats. Brain Res 2013, 1499:43-52.

79. Meck WH: Neuroanatomical localization of an internal clock: a functional link between mesolimbic, nigrostriatal, and mesocortical dopaminergic systems. Brain Res 2006 1109:93-107.

80. Wiener M, Turkeltaub $\mathrm{P}$, Coslett HB: The image of time: a voxel-wise meta-analysis. Neurolmage 2010, 49:1728-1740.

81. Pfeuty $M$, Dilharreguy $B$, Gerlier L, Allard M: fMRI identifies the right inferior frontal cortex as the brain region where time interval processing is altered by negative emotional arousal. Hum Brain Map 2015, 36:981-995.

82. van Wassenhove V, Wittmann M, Craig, AD, Paulus MP: Psychological and neural 
mechanisms of subjective time dilation. Front Neurosci 2011, 5:56.

83. Koubeissi MZ, Bartolomei F, Beltagy A, Picard F: Electrical stimulation of a small brain area reversibly disrupts consciousness. Epilepsy Beh 2014, 37:32-35.

84. Wittmann M: Modulations of the experience of self and time. Conscious Cogn 2015, 38:172-181.

85. Buhusi CV, Meck WH: Relativity theory and time perception: single or multiple clocks? PLOS ONE 2009, 4(7): e6268.

86. Buhusi $\mathrm{CV}$, Meck $\mathrm{WH}$ : Relative time sharing: new findings and an extension of the resource allocation model of temporal processing. Philos Trans $R$ Soc Lond B, 2009, 364:1875-1885.

87. Meck WH, MacDonald CJ: Amygdala inactivation reverses fear's ability to impair divided attention and make time stand still. Behav Neurosci 2007,121:707-720.

88. Farrell MS: Using DREADDs to isolate internal clocks. Front Integr Neurosci 2011, 5:87.

89. Narayanan NS, Land BB, Solder JE, Deisseroth K, DiLeone RJ: Prefrontal D1 dopamine signaling is required for temporal control. Proc Natl Acad Sci USA 2012, 109:2072620731.

90. Smythies J, Edelstein L, Ramachandran VS: The claustrum: structural, functional, and clinical neuroscience. Academic Press, 2014.

91. Pöppel E: Pre-semantically defined temporal windows for cognitive processing. Phil Trans $R$ Soc B 2009, 364:1887-1896.

92. Sitt JD, King J-R, Naccache L, Dehaene S: Ripples of consciousness. Trends Cogn Sci 2013, 17:552-554.

93. Nyberg L, Kim ASN, Habib R, Levine B, Tulving E: Consciousness of subjective time in the brain. Proc Nat Acad Sci USA 2010, 107:22356-22359.

94. Stiefel KM, Merrifield A, Holcombe AO: The claustrum's proposed role in consciousness is supported by the effect and target localization of Salvia divinorum. Front Integr Neurosci 2014, 8:20.

95. Craig $A D$ : Emotional moments across time: a possible neural basis for time perception in the anterior insula. Phil Trans $R$ Soc $B$ 2009, 364:1933-1942.

96. Yin $B$, Troger A: Exploring the $4^{\text {th }}$ dimension: hippocampus, time, and memory revisted. Front Integr Neurosci 2011, 5:36.

97. Merchant $\mathrm{H}$, Harrington DL, Meck WH: Neural basis of the perception and estimation of

•• time. Annu Rev Neurosci 2013, 36:313-336.

A "state-of-the-art" review of the cortico-thalamic-basal ganglia (CTBG) circuits underlying interval timing in the hundredths of milliseconds-to-minutes range. Taken together, the data suggest that a core timing mechanism in the motor system interacts with context-dependent sensory areas distributed across the cortex. The findings from a large number of studies are interpreted within the context of the striatal beat frequency model of interval timing as originally described by Matell and Meck [49]. 


\section{Figure captions.}

Figure 1. Input-output connectivity of the claustrum. The architecture of the input-output connectivity of the claustrum is charted in sagittal (A) and coronal (B) sections of the rodent brain. The primary inputs to the claustrum are from the cortical modalities, which display symmetrical reciprocal connections. Additional inputs come from the prefrontal cortex as well as subcortical inputs from the striatum, thalamus, amygdala, and dorsal raphe. Abbreviations: PrL, prelimbic cortex; ACA, anterior cingulate area; ILA, infralimbic area; MO, motor cortex; SS, somatosensory cortex; ENT, entorhinal area; AI, agranular insular area; PIR, piriform area; PrL, prelimbic area; Fr, frontal cortex; AUD, auditory cortex; VIS, visual cortex; STR, striatum; TH, thalamus; AMG, amygdala; HPC, hippocampus; DR, dorsal raphe. Coronal (C) section of the human brain showing the cortico-claustral medial pathway spreading between claustrum and basal ganglia using constrained spherical deconvolution tractography to map white matter fibers. Phylogenetic differences in the organization of the sensory systems of different species makes it difficult to identify homologous areas, consequently these illustrations should be taken as a general outline. Adapted from $[62 \bullet \bullet, 74,90]$.

Figure 2. A unified model of interval timing. The striatal network (b/ue) and the olivocerebellar network (green) are connected to each other via multiple loops, and with the thalamus, preSMA/SMA, and the cerebral cortex. Separate bi-directional circuits connect the claustrum to the cortex and the striatum. Dopaminergic pathways are shown in orange, inhibitory projections in blue, excitatory projections in red, and known anatomical connections in solid lines, respectively. Abbreviations: GPe, globus pallidus external; GPi, globus pallidus internal; IO, inferior olive; SMA, supplementary motor area; SNpc, substantia nigra pars compacta; SNpr, substantia nigra pars reticulate; STN, subthalamic nucleus; VTA, ventral tegmental area. Adapted from [20, 57, 97••]. 
Figure 1.

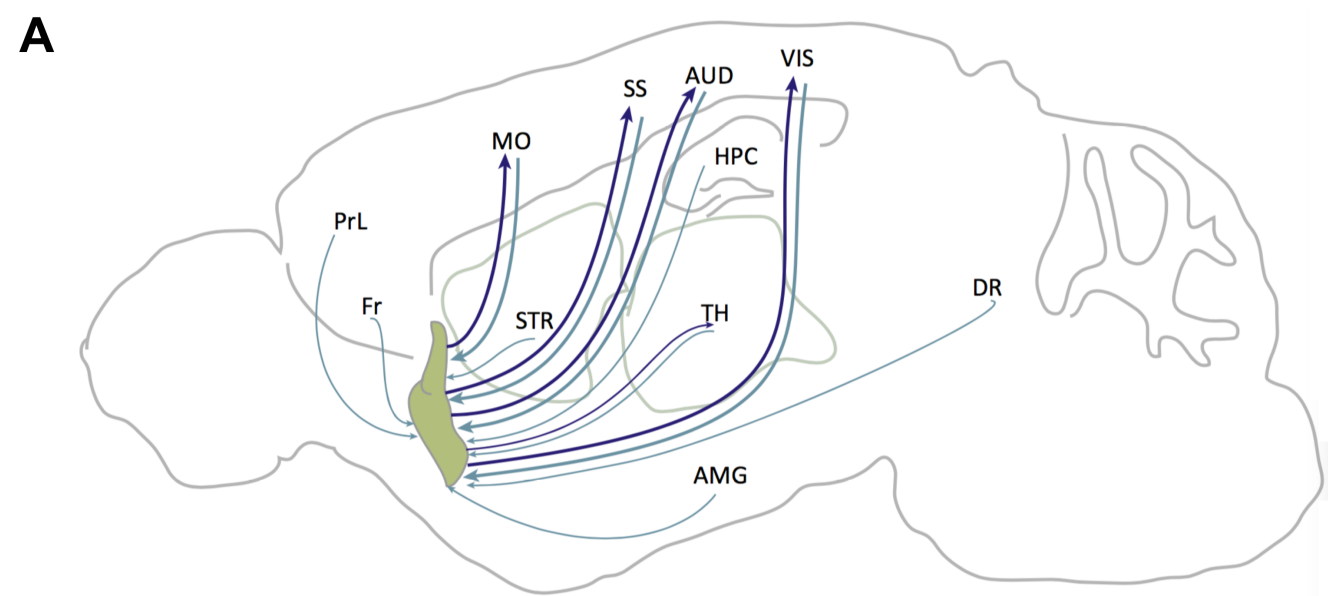

B

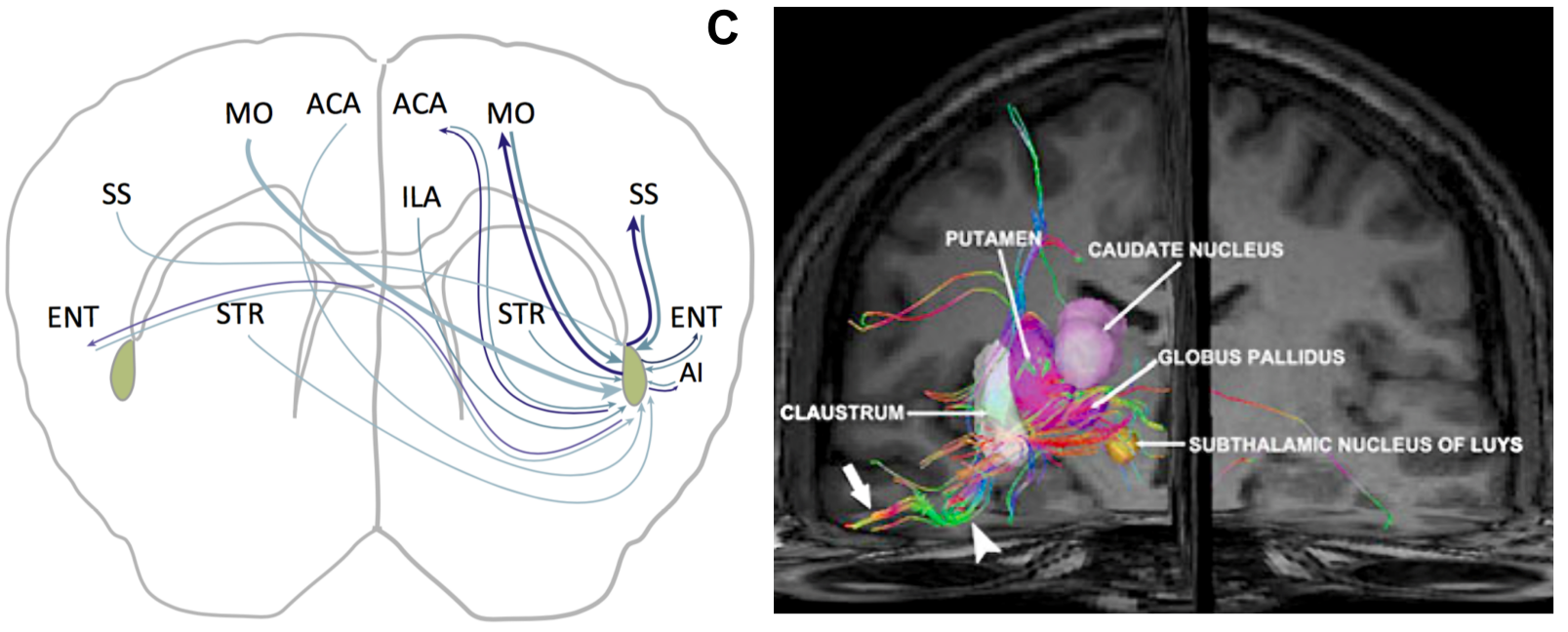


Figure 2.

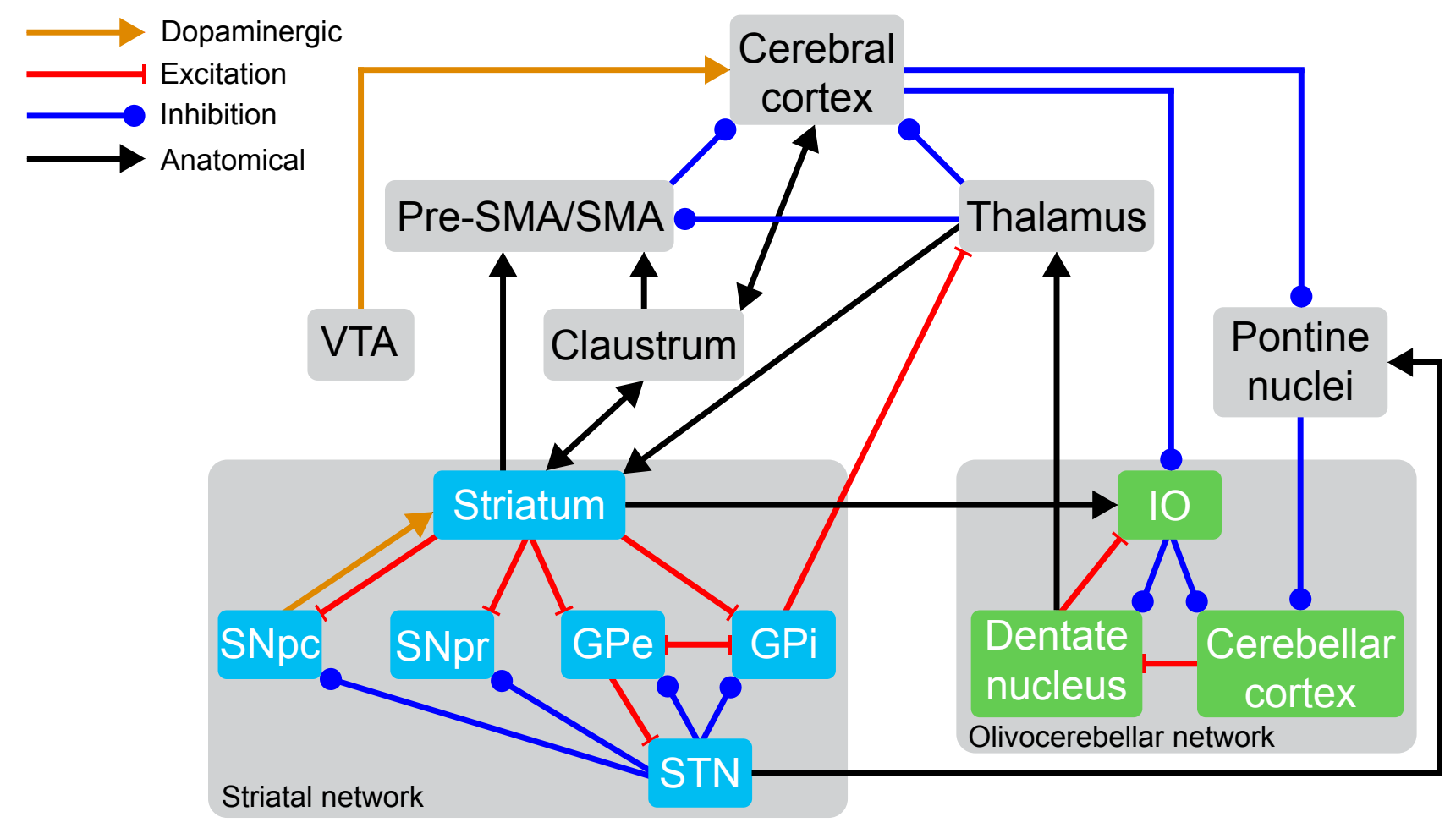

